\title{
Vanishing Thrombus Resulting in Pulmonary Saddle Embolism
}

Oriowo Babatunde ${ }^{1}$, Renee Mong ${ }^{2}$ Elizabeth Kudlaty ${ }^{1}$ and Bhagwan Satiani ${ }^{1,2^{*}}$

${ }^{1}$ Division of Vascular Diseases \& Surgery, The Ohio State University, Columbus, Ohio, USA

${ }^{2}$ Division of Vascular Laboratory Heart \& Vascular Center, The Ohio State University, Columbus, Ohio, USA

"Corresponding author: Bhagwan Satiani, Division of Vascular Diseases \& Surgery, Department of Surgery, College of Medicine, The Ohio State University, Columbus, Ohio 43210, USA, Tel: 614293 8536; E-mail: bhagwan.satiani@osumc.edu

Rec date: Nov 27, 2014; Acc Date: Dec 06, 2014; Pub Date: Dec 08, 2014

Copyright: (c) 2015 Babatunde O, et al. This is an open-access article distributed under the terms of the Creative Commons Attribution License, which permits unrestricted use, distribution, and reproduction in any medium, provided the original author and source are credited.

\section{Abstract}

While there are rare reports of pulmonary embolism in patients with acute deep venous thrombosis following duplex venous scanning, it is distinctly rare to document a case of pulmonary embolism during the scan. This case demonstrates the challenge for the technologist and reading physician as well as possible preventive measures to be utilized during scanning in patients with acute deep venous thrombosis.

Keywords: Deep vein thrombosis; Pulmonary embolism; Vanishing thrombus

\section{Introduction}

The precise number of people affected by acute Deep Venous Thrombosis (DVT) and /Pulmonary Embolism (PE) is unknown, but estimates range from 300,000 to 600,000 ( 1 to 2 per 1,000 , and in those over 80 years of age, as high as 1 in 100) each year in the United States [1]. Duplex Venous Scanning (DVS) has become the standard test for the diagnosis of DVT. The accuracy of DVS is excellent for above knee DVT although it is less accurate for calf DVT [2]. Most thrombi detected by DVS are fairly well attached to the vein wall and stable. There are reports in the literature of 'free floating' thrombi that later propagate and cause pulmonary embolism $[3,4]$. Even with this entity, since DVS is not three dimensional, the label of 'free floating' is somewhat controversial. However, there are few published reports of a well visualized deep vein thrombus on DVS, which then 'vanished' either during the same examination or soon thereafter followed by a documented pulmonary embolus.

\section{Case History}

We report the case of a 53 year old gentleman with a middle cerebral artery distribution stroke with hemorrhagic conversion and cerebral edema and status post craniectomy and residual spastic right hemiparesis CVA. Who had recently suffered an ischemic stroke with hemorrhagic conversion. He had convalesced through his illness and was recovering at an inpatient rehabilitation facility. During the course of his rehabilitation he was found to have a right peroneal deep vein thrombus on screening DVS. In light of his recent hemorrhagic stroke he was managed only with subcutaneous heparin and serial DVS exams. Though he exhibited no limb swelling on daily physical exam at the time of his next serial exam, the sonographer detected a right femoral vein thrombus. The sonographer then examined the contralateral limb and decided to re-image the right limb. On repeat imaging of the right femoral vein the previously seen thrombus was not seen. The sonographer then notified the referring physician who obtained a Computed Tomographic scan (CT) on the asymptomatic patient, which demonstrated a pulmonary saddle embolus. The patient was then permitted by Neurosurgery to be treated with therapeutic heparin. He also underwent placement of an Inferior Vena Cava (IVC filter). The patient has since done well on anticoagulation with no recurrence.

\section{Discussion}

The clinical entity of the 'vanishing' thrombus is underreported in the vascular literature so that a consensus regarding any preventive or therapeutic measures is hard to find. In informal electronic communications with several medical directors of busy vascular laboratories, almost all remembered a case they had seen during their professional careers. There are however, very few published reports $[3,4]$.

There are reports of free floating thrombi and thrombi developing after intervention resulting in pulmonary embolism, but a true vanishing thrombus, by definition is one that disappears by the time of completion of a standard lower extremity venous Doppler exam, is indeed a rare entity [5].

There are several circumstances where a previously visualized acute venous thrombus seen on DVS is noted to have 'vanished' on repeat DVS $[5,6]$. One scenario is where an acute thrombus disappears during the examination often with compression [5,7]. A PE has also been reported after laser ablation of the great saphenous vein [8]. We have previous experience with a very small thrombus in the common femoral vein which disappeared with slight compression. A CT scan was subsequently negative. Another scenario is one we have described where for some reason, the sonographer decided to re-check the affected limb for confirmation and noticed the absence of the thrombus. Yet another setting is when the patient is either asymptomatic or symptomatic after a DVS showed acute DVT and a repeat scan several hours or days later showed that the thrombus had disappeared.

The question then arises, what do we do when faced with such a situation in terms of prevention and treatment? This finding on DVS examination often leads to confusion on the part of the vascular sonographer and the physician reading the study. In our case the patient had a CT PE study as a result of the finding. 
Page 2 of 2

Are there steps a sonographer can take to prevent a PE possibly due to the examination technique? With millions of DVS examinations performed and so few reports of a PE following the test, there is no clear evidence that any particular technique leads to the thrombus dislodging from its bed. There is no clear evidence that there are certain features of thrombi that can alert the technologist and interpreting physician to the impending danger of a PE. Should the way DVS examinations are being performed be changed? Should color Doppler be utilized from the beginning of the study? Our study protocol begins with transverse grayscale images with and without compressions starting at the common femoral vein followed by spectral Doppler at the ipsilateral common femoral, femoral and popliteal veins and the contralateral common femoral vein. Color flow is turned on to expedite identification of the veins as well as adequacy of complete filling of each vein examined. In general, if poorly attached thrombus is noted at any level, the sonographer is cautious about full compression.

Much like the so called 'free floating thrombus' which itself is controversial as far an indication for IVC filter placement, does the identification of a vanishing thrombus demand immediate anticoagulation and filter placement? There is some controversy about the incidence of PE being greater in patients who have 'free floating' thrombus compared to those who do not by DVS. Some reports in the literature with a relatively small number of patients indicate the risk of $\mathrm{PE}$ with documentation of free floating thrombus is similar to patients with occlusive thrombus as confirmed by DVS, and further that the majority of PE in such patients are documented prior to positive DVT study $[3,4]$. Therefore, it remains unclear the specific characteristics that lead some free floating thrombus to embolize. In the case discussed, the sonographer had already completed the assessment of the right femoral vein, had already identified the thrombus and in reality did not need to repeat that examination but decided to re- confirm the finding. Given the potentially fatal complication if such a thrombus progressed to a pulmonary embolus as in the case described, necessary protocols should be in place to pick up more of these elusive thrombi. Should anticoagulation be initiated prior to completion of a positive study?

Further reporting of this interesting phenomenon is required before such recommendations can be taken seriously though.

\section{References}

1. Centers for disease control and prevention (2014) Deep Vein Thrombosis (DVT) / Pulmonary Embolism (PE) - Blood Clot Forming in a Vein.

2. Forbes K, Stevenson AJ (1998) The use of power doppler ultrasound in the diagnosis of isolated deep venous thrombosis of the calf. Clin Radiol 53: 752-754.

3. Baldridge ED, Martin MM, Welling RE (1990) Clinical significance of the free-floating venous thrombi. J Vasc Surg 11: 62-67.

4. Pacouret G, Alison D, Pottier JM, Bertrand P, Charbonnier B (1997) Free-floating thrombus and embolic risk in patients with angiographically confirmed proximal deep venous thrombosis. A prospective study. Arch Intern Med 157(3):305-308.

5. Ferral H, Yedlicka JW Jr, Hunter DW, Letourneau JG (1992) Pulmonary embolism during compression US of the lower extremity Radiology 185 : 614.

6. Sufian S, Arnez A, Lakhanpal S (2012) Case of the disappearing heatinduced thrombus causing pulmonary embolism during ultrasound evaluation. J Vasc Surg 55: 529-531.

7. Perlin SJ (1992) Pulmonary embolism during compression US of the lower extremity. Radiology 184: 165-166.

8. Rosales-Velderrain A, Gloviczki P, Said SM, Hernandez MT, Canton LG, et al. (2013) Pulmonary embolism after endovenous thermal ablation of the saphenous vein. Semin Vasc Surg. 26: 14-22. 